\title{
Erratum: Hybrid quantum repeater based on resonant qubit-field interactions [Phys. Rev. A 96, 052329 (2017)]
}

\author{
József Zsolt Bernád
}

(Received 25 May 2018; published 12 June 2018)

DOI: 10.1103/PhysRevA.97.069903

Equation (14) should read

$$
\begin{aligned}
\hat{\rho}_{A B} & =\frac{(1+x)\left|\Psi^{-}\right\rangle\left\langle\Psi^{-}|+(1-x)| \Phi_{\phi}^{-}\right\rangle\left\langle\Phi_{\phi}^{-}|+i y| \Phi_{\phi}^{-}\right\rangle\left\langle\Psi^{-}|-i y| \Psi^{-}\right\rangle\left\langle\Phi_{\phi}^{-}\right|}{2}, \\
x & =\exp \left\{-\bar{n}[1-\cos (2 \varphi)]\left(1-\eta e^{-\gamma T}\right)\right\} \cos \left[\bar{n} \sin (2 \varphi)\left(1-\eta e^{-\gamma T}\right)\right], \\
y & =\exp \left\{-\bar{n}[1-\cos (2 \varphi)]\left(1-\eta e^{-\gamma T}\right)\right\} \sin \left[\bar{n} \sin (2 \varphi)\left(1-\eta e^{-\gamma T}\right)\right],
\end{aligned}
$$

where the error came from a simple algebraic mistake. However, it does not affect the success probability $P_{\text {Gen }}$, which remains equal to 0.5. The concurrence of the state in Eq. (1) is now $\sqrt{x^{2}+y^{2}}$.

Substituting the corrected state into the purification protocol of Sec. II B, we get

$$
\hat{\rho}_{A_{1}, B_{1}}^{(1)}=\frac{(1+x)^{2}}{2+2 x^{2}}\left|\Psi^{-}\right\rangle\left\langle\Psi^{-}\left|+\frac{(1-x)^{2}}{2+2 x^{2}}\right| \Psi^{+}\right\rangle\left\langle\Psi^{+}\left|+\frac{y^{2}}{2+2 x^{2}}\right| \Psi^{-}\right\rangle\left\langle\Psi^{+}\left|+\frac{y^{2}}{2+2 x^{2}}\right| \Psi^{+}\right\rangle\left\langle\Psi^{-}\right|,
$$

with a success probability of $\left(1+x^{2}\right) / 4$. After repeating the protocol for $N$ times we obtain

$$
\hat{\rho}^{(N)}=f_{(N)}(x)\left|\Psi^{-}\right\rangle\left\langle\Psi^{-}\left|+g_{(N)}(x)\right| \Psi^{+}\right\rangle\left\langle\Psi^{+}\right|+h_{(N)}(x)\left(\left|\Psi^{-}\right\rangle\left\langle\Psi^{+}|+| \Psi^{+}\right\rangle\left\langle\Psi^{-}\right|\right),
$$

with success probability,

$$
\begin{aligned}
P_{\text {Pur }} & =\left[P_{(0)}\right]^{2^{N-1}}\left[P_{(1)}\right]^{2^{N-2}} \cdots\left[P_{(N-2)}\right]^{2} P_{(N-1)}, \\
P_{(k)} & =\frac{f_{(k)}^{2}(x)+g_{(k)}^{2}(x)}{2}
\end{aligned}
$$

where

$$
\begin{array}{cc}
f_{(k+1)}(x)=\frac{f_{(k)}^{2}(x)}{f_{(k)}^{2}(x)+g_{(k)}^{2}(x)}, & f_{(0)}(x)=\frac{1+x}{2}, \\
g_{(k+1)}(x)=\frac{g_{(k)}^{2}(x)}{f_{(k)}^{2}(x)+g_{(k)}^{2}(x)}, & g_{(0)}(x)=\frac{1-x}{2}, \\
h_{(k+1)}(x)=\frac{h_{(k)}^{2}(x)}{f_{(k)}^{2}(x)+g_{(k)}^{2}(x)}, & h_{(1)}(x)=\frac{y^{2}}{2+2 x^{2}} .
\end{array}
$$

In general, $h_{(N)}(x)$ tends to zero as $N$ increases and stays constant at the value of 0.5 only when $y=1$. However, when $y=1$ then $x=0$, which means that the state in Eq. (1) is not purifiable.

Equations (2)-(4) are the same as in the published version. Therefore, the main results on the repeater rates are unaffected, and all conclusions of the paper remain unchanged. 